\title{
Tracking/Robust Trade-off Design of a Sampled-data PID Controller for Second-order Plus Dead-time Systems
}

\author{
Ryo Kurokawa and Takao Sato \\ Department of Mechanical Engineering, University of Hyogo \\ 2167 Shosha, Himeji, Hyogo 671-2280 Japan \\ E-mail: tsato@eng.u-hyogo.ac.jp \\ Ramon Vilanova \\ Department of Telecommunications and Systems Engineering, Universitat Autònomade Barcelona \\ Edifici Q-Campus de la UAB, 08193 Bellaterra, Barcelona, Spain \\ E-mail: ramon.vilanova@uab.cat \\ Yasuo Konishi \\ Department of Mechanical Engineering, University of Hyogo \\ 2167 Shosha, Himeji, Hyogo 671-2280 Japan \\ E-mail:konishi@eng.u-hyogo.ac.jp
}

\begin{abstract}
In this paper, we propose a new design method of a second-order plus dead-time (SOPDT) sampled-data ProportionalIntegral-Derivative (PID) control system, where the continuous-time plant is controlled using the discrete-time controller. The proposed control system is designed so that the tracking performance is optimized subject to the stability margin constraint. In the present study, the servo and regulation optimal controllers are designed. Finally, the effectiveness of the proposed method is demonstrated through numerical examples.
\end{abstract}

Keywords: PID control, Sampled-data system, SOPDT system, Sensitivity function, Robust

\section{Introduction}

Proportional-Integral-Derivative (PID) ${ }^{1,2}$ control has been widely used in industry. Since the performance of PID control depends on the tuning parameters, additional tuning methods have been studied recently. Although the stability of a control system is critical, its tracking performance is also important. However, because of the trade-off relationship between stability and tracking performance, they cannot be optimized simultaneously. Arrieta and Vilanova ${ }^{3,4}$ proposed a simple PID tuning method that optimizes the tracking performance subject to a prescribed robust stability. In this method, the optimal PID parameters are decided based on a first-order plus dead-time (FOPDT) continuous-time system. In order to design a discrete-time control system, Tajika et al..$^{5}$ proposed a design method for controlling a discretetime FOPDT system. The present study discusses a design method of the PID controller for controlling a second-order plus dead-time (SOPDT) system, in which the continuous-time plant is controlled using the discretetime controller. In the proposed method, both servo and regulation optimized control methods are designed. 
Finally, the effectiveness of the proposed method is demonstrated through numerical examples.

\section{Description of the Control System}

Consider the continuous-time controlled plant given as follows:

$$
P(s)=\frac{K \omega_{n}^{2}}{s^{2}+2 \zeta \omega_{n} s+\omega_{n}^{2}} e^{-L s}
$$

where $K$ is the plant gain, $\omega_{n}$ is the natural angular frequency, $\zeta$ is the damping coefficient, and $L$ is the dead-time. In the present study, we discuss the design method of the sampled-data control system using the following discrete-time PID control law:

$$
\begin{gathered}
u(k)=C_{e}\left(z^{-1}\right) e(k)+C_{y}\left(z^{-1}\right) y(k) \\
C_{d}\left(z^{-1}\right)=C_{e}\left(z^{-1}\right)+C_{y}\left(z^{-1}\right) \\
C_{e}\left(z^{-1}\right)=K_{p}\left\{1+\frac{T_{s}}{T_{i}\left(1-z^{-1}\right)}\right\} \\
C_{y}\left(z^{-1}\right)=K_{p}\left\{\frac{T_{d}\left(1-z^{-1}\right)}{T_{s}}\right\}
\end{gathered}
$$

where $u(k)$ is the control input, $y(k)$ is the plant output, $e(k)(=r(k)-y(k))$ is the control error, and $r(k)$ is the reference. Moreover, $T_{s}, K_{p}, T_{i}$, and $T_{d}$ are the sampling time, the proportional gain, the integral time, and the differential time, respectively.

\section{Definition of the Optimization Problem}

As the constraint condition, the stability margin is defined using the sensitivity function, and the evaluation function for the tracking performance is also defined.

\subsection{Constraint condition}

The sensitivity function $S_{f}\left(z^{-1}\right)$ is defined as follows:

$$
S_{f}\left(z^{-1}\right)=\frac{1}{1+C_{d}\left(z^{-1}\right) P_{d}\left(z^{-1}\right)}
$$

where $P_{d}\left(z^{-1}\right)$ is the discrete-time controlled plant. Using the sensitivity function, the constraint condition is defined as follows:

$$
\begin{gathered}
\left|M_{s}-M_{s}^{d}\right|=0 \\
M_{s}=\max _{\omega}\left|S_{f}\left(e^{-j \omega}\right)\right|
\end{gathered}
$$

where $M_{\mathrm{s}}$ is the maximum value of the sensitivity function, and $M_{S}^{d}$ is the desired value selected by the designer. The recommended range of $M_{s}^{d}$ is from 1.4 to $2.0^{1}$. The smaller the value of $M_{s}$, the larger the stability margin. On the other hand, the larger the value of $M_{s}$, the better the tracking performance, although the stability margin becomes small.

\subsection{Evaluation function}

In the present study, the evaluation function $J$ is defined as the integral absolute error:

$$
J=\sum_{k=0}^{\infty}|e(k)|=\sum_{k=0}^{\infty}|r(k)-y(k)|
$$

A trade-off relationship exists between the servo performance and the regulation performance. In the present study, the PID parameters are optimized for the servo and regulation control, respectively.

\section{Controller Design}

The PID parameters are optimized for a normalized system, and hence, dimensionless parameters are defined as $\tau=L \omega_{n}, h=T_{s} \omega_{n}, \kappa_{p}=K_{p} K, \tau_{i}=T_{i} \omega_{n}$, and $\tau_{d}=T_{d} \omega_{n}$. The range of these parameters are set as $0.1 \leq \tau \leq 1.0,0.01 \leq h \leq 0.10$, and $0.3 \leq \zeta \leq 1.2$. In the proposed method, the constrained optimal problem is preliminarily solved for a designated finite plant, which is defined by discrete $\tau, h$, and $\zeta$, and the data set in which the optimal normalized PID parameters for discrete $\tau, h$, and $\zeta$, is obtained. In Fig. 1, the obtained normalized PID parameters are plotted by ${ }^{\circ}$, where $M_{s}^{d}=1.4$ and $T_{s}=0.01$. 

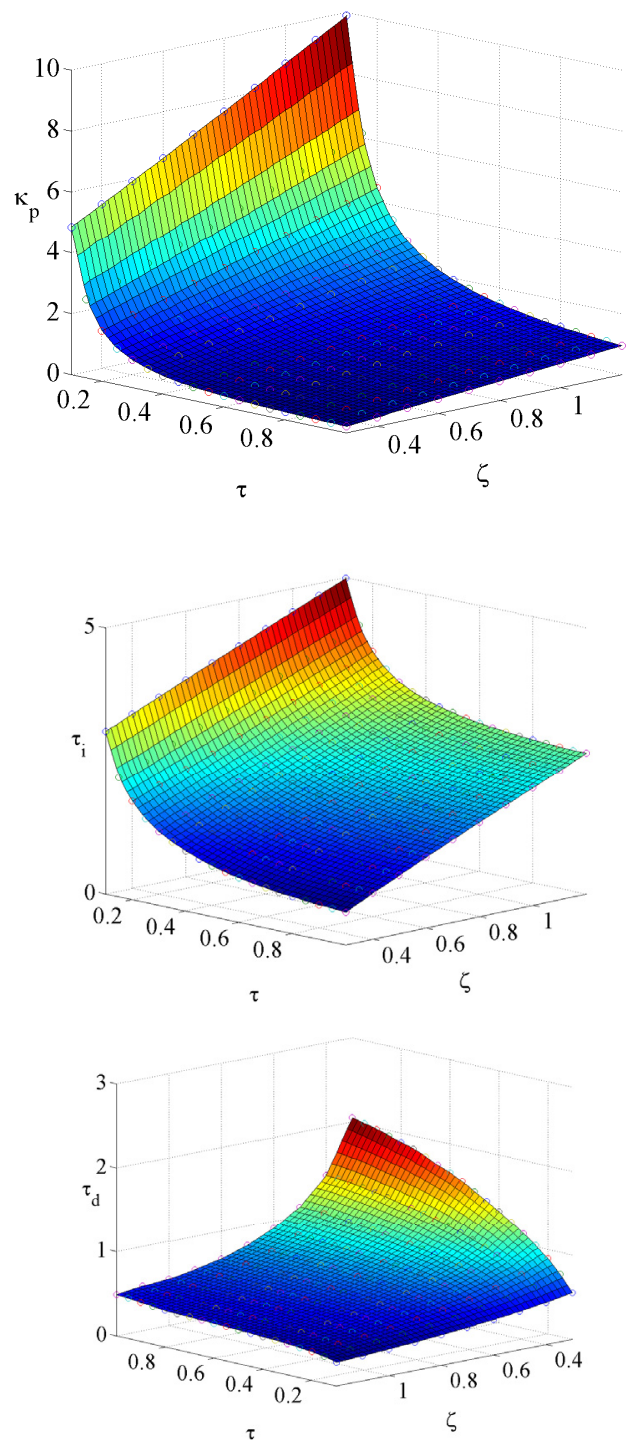

Fig. 1. Relationships among $\tau, \zeta$, and $\kappa_{p}, \tau_{i}$ and $\tau_{d}$ (servo design, $M_{S}^{d}=1.4$ and $T_{s}=0.01$ )

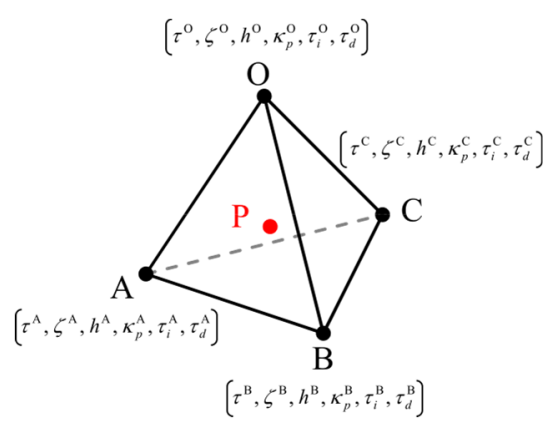

Fig. 2. Image of the linear interpolation
The desired normalized PID parameters for an arbitrary plant are decided by the linear interpolation from the data set. Practically speaking, the interpolated parameters are calculated using the nearest four points, as shown in Fig. 2. From this figure, the vector equation is obtained as follows:

$$
\begin{gathered}
\overrightarrow{\mathrm{OP}}=\alpha \overrightarrow{\mathrm{OA}}+\beta \overrightarrow{\mathrm{OB}}+\gamma \overrightarrow{\mathrm{OC}} \\
0 \leq \alpha \leq 1 \\
0 \leq \beta \leq 1 \\
0 \leq \gamma \leq 1
\end{gathered}
$$

where point $\mathrm{O}\left[\tau^{\mathrm{O}}, \zeta^{\mathrm{O}}, h^{\mathrm{O}}, \kappa_{p}^{\mathrm{O}}, \tau_{i}^{\mathrm{O}}, \tau_{d}^{\mathrm{O}}\right], \mathrm{A}\left[\tau^{\mathrm{A}}, \zeta^{\mathrm{A}}\right.$, $\left.h^{\mathrm{A}}, \kappa_{p}^{\mathrm{A}}, \tau_{i}^{\mathrm{A}}, \tau_{d}^{\mathrm{A}}\right], \mathrm{B}\left[\tau^{\mathrm{B}}, \zeta^{\mathrm{B}}, h^{\mathrm{B}}, \kappa_{p}^{\mathrm{B}}, \tau_{i}^{\mathrm{B}}, \tau_{d}^{\mathrm{B}}\right]$, and $\mathrm{C}$ $\left[\tau^{\mathrm{C}}, \zeta^{\mathrm{C}}, h^{\mathrm{C}}, \kappa_{p}^{\mathrm{C}}, \tau_{i}^{\mathrm{C}}, \tau_{d}^{\mathrm{C}}\right]$ are the nearest points of the desired $\left[\tau^{\mathrm{P}}, \zeta^{\mathrm{P}}, h^{\mathrm{P}}\right]$. Then, Eq. (6) is rearranged as follows:

$$
\begin{gathered}
\kappa_{p}^{\mathrm{P}}=\kappa_{p}^{\mathrm{O}}+\alpha\left(\kappa_{p}^{\mathrm{A}}-\kappa_{p}^{\mathrm{O}}\right)+\beta\left(\kappa_{p}^{\mathrm{B}}-\kappa_{p}^{\mathrm{O}}\right)+\gamma\left(\kappa_{p}^{\mathrm{C}}-\kappa_{p}^{\mathrm{O}}\right) \\
\tau_{i}^{\mathrm{P}}=\tau_{i}^{\mathrm{O}}+\alpha\left(\tau_{i}^{\mathrm{A}}-\tau_{i}^{\mathrm{O}}\right)+\beta\left(\tau_{i}^{\mathrm{B}}-\tau_{i}^{\mathrm{O}}\right)+\gamma\left(\tau_{i}^{\mathrm{C}}-\tau_{i}^{\mathrm{O}}\right) \\
\tau_{d}^{\mathrm{P}}=\tau_{d}^{\mathrm{O}}+\alpha\left(\tau_{d}^{\mathrm{A}}-\tau_{d}^{\mathrm{O}}\right)+\beta\left(\tau_{d}^{\mathrm{B}}-\tau_{d}^{\mathrm{O}}\right)+\gamma\left(\tau_{d}^{\mathrm{C}}-\tau_{d}^{\mathrm{O}}\right)
\end{gathered}
$$

Solving these equations, the desired $\kappa_{p}^{\mathrm{P}}, \tau_{i}^{\mathrm{P}}$, and $\tau_{d}^{\mathrm{P}}$ for $\left[\tau^{\mathrm{P}}, \zeta^{\mathrm{P}}, h^{\mathrm{P}}\right]$ are obtained, where $\alpha, \beta$, and $\gamma$ are decided based on the following equations:

$$
\begin{gathered}
\tau^{\mathrm{P}}-\tau^{\mathrm{O}}=\alpha\left(\tau^{\mathrm{A}}-\tau^{\mathrm{O}}\right)+\beta\left(\tau^{\mathrm{B}}-\tau^{\mathrm{O}}\right)+\gamma\left(\tau^{\mathrm{C}}-\tau^{\mathrm{O}}\right) \\
\zeta^{\mathrm{P}}-\zeta^{\mathrm{O}}=\alpha\left(\zeta^{\mathrm{A}}-\zeta^{\mathrm{O}}\right)+\beta\left(\zeta^{\mathrm{B}}-\zeta^{\mathrm{O}}\right)+\gamma\left(\zeta^{\mathrm{C}}-\zeta^{\mathrm{O}}\right) \\
h^{\mathrm{P}}-h^{\mathrm{O}}=\alpha\left(h^{\mathrm{A}}-h^{\mathrm{O}}\right)+\beta\left(h^{\mathrm{B}}-h^{\mathrm{O}}\right)+\gamma\left(h^{\mathrm{C}}-h^{\mathrm{O}}\right)
\end{gathered}
$$

In Fig. 1, the interpolated parameters are plotted over the discrete calculated optimal parameters. Furthermore, $M_{S}$ is calculated for both the preliminarily solved and interpolated systems using the approximation method, and the obtained $M_{S}$ values are shown in Table 1. This result reveals that the proposed decision method is sufficiently effective.

Table 1. Obtained $M_{S}$

\begin{tabular}{|c|c|c|c|c|c|c|}
\hline & \multicolumn{3}{|c|}{ Servo design } & \multicolumn{3}{c|}{ Regulation design } \\
\hline$M_{S}^{d}$ & Min & Mean & Max & Min & Mean & Max \\
\hline 1.4 & 1.398 & 1.403 & 1.440 & 1.399 & 1.403 & 1.453 \\
\hline 1.6 & 1.599 & 1.605 & 1.668 & 1.597 & 1.605 & 1.663 \\
\hline 1.8 & 1.790 & 1.807 & 1.909 & 1.798 & 1.807 & 1.897 \\
\hline 2.0 & 1.996 & 2.010 & 2.156 & 1.997 & 2.009 & 2.137 \\
\hline
\end{tabular}




\section{Numerical Simulation}

In this section, the effectiveness of the proposed method is confirmed.

\subsection{Control performance for various values of $\zeta$}

First, the control performance is confirmed for $\zeta$. The controlled plant is defined as $K=4.2, \omega_{n}=1.13$, and $L=0.44$ in Eq. (1), and $T_{S}=0.018$. Here, we consider four pattern damping coefficients: $\zeta^{1}=0.451, \zeta^{2}=$ $0.69, \zeta^{3}=1.0$, and $\zeta^{4}=1.199$. The control results are shown in Fig. 3. The reference value is set to 1.0, and the unit step disturbance signal is added after $20 \mathrm{~s}$. Figure 3 shows that the proposed method is effective for underand over-damping systems.

\subsection{Verification of stability margin}

Next, the stability margin is confirmed. Here, the controlled plant is defined as $K=2.02, \omega_{n}=0.91$, $\zeta=0.33$, and $L=0.98$ in Eq. (1), and $T_{s}=0.05$. After $40 \mathrm{~s}$, the dynamics is changed to $K=2.6, \omega_{n}=$

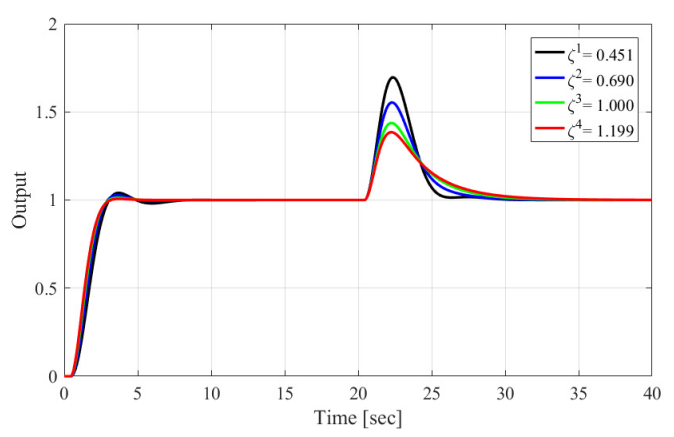

Fig. 3. Output responses for each damping coefficient $\zeta^{i}$ (servo design and $M_{S}^{d}=1.4$ )

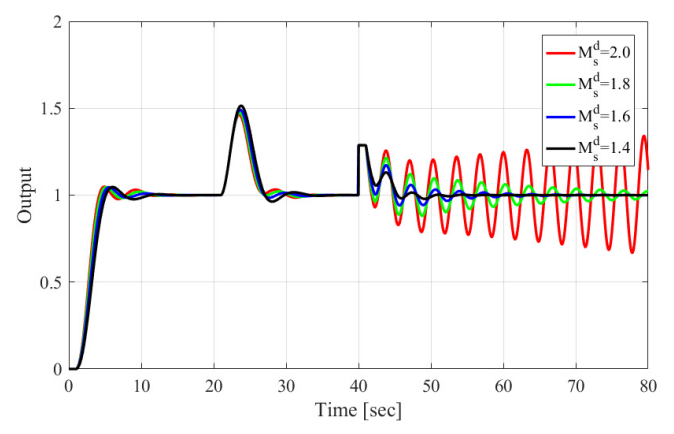

Fig. 4. Output responses for each $M_{s}^{d}$
1.3, $\zeta=0.43$, and $L=0.43$ as the model variation. Furthermore, $M_{s}^{d}$ is varied as 1.4, 1.6, 1.8, and 2.0, respectively, and the control results are compared. The obtained results are shown in Fig. 4. The reference value is 1.0, and the unit step disturbance signal is added after $20 \mathrm{~s}$. The model variation is caused at $40 \mathrm{~s}$. Figure 3 shows that the smaller the value of $M_{s}^{d}$, the larger the stability margin, and vice versa. On the other hand, the larger the value of $M_{s}^{d}$, the better the tracking performance, and vice versa.

\section{Conclusion}

In the present study, we have proposed a new design method for controlling an SOPDT sampled-data system, where the continuous-time plant is controlled by the discrete-time PID control law. In the proposed method, the PID parameters are designed for the normalized system, and the tracking performance is optimized subject to the assigned $M_{s}^{d}$. Finally, the effectiveness of the proposed method is demonstrated through numerical examples.

\section{Acknowledgements}

The present study was supported by JSPS KAKENHI Grant Number JP16K06425. The authors would like to express their sincere thanks for the support from the Japan Society for the Promotion of Science.

\section{References}

1. K. J. Åström and T. Hägglund, Advanced PID Control, ISA-Instrumentation, Systems, and Automation Society, 2006.

2. R. Vilanova and A. Visioli, PID Control in the Third Millennium (Springer 2012, UK).

3. O. Arrieta and R. Vilanova, Simple PID Tuning Rules with Guaranteed $M_{s}$ Robustness Achievement, IFAC Proceedings 44(1), 2011, pp. 12042-1204.

4. O. Arrieta and R. Vilanova, Simple Servo/Regulation Proportional-Integral-Derivative (PID) Tuning Rules for Arbitrary $M_{s}$-Based Robustness Achievement, Industrial \& Engineering Chemistry Research 51(6), 2012, pp. 2666-2674.

5. H. Tajika, T. Sato, R. Vilanova and Y. Konishi, Optimal PID Control in Discrete Time Using a Sensitivity Function, 23th Mediterranean Conference on Control and Automation (MED), 2015, pp. 249-254. 